\title{
Una Aproximación Tafonómica a los Restos de Peces en Laguna Llancanelo (Mendoza)
}

\author{
A Taphonomic Approach to Fish Remains at Llancanelo Lagoon (Mendoza)
}

Mercedes Corbat ${ }^{\mathrm{i}}$

\section{RESUMEN}

El origen de las acumulaciones de restos de peces en contextos arqueológicos de ambientes lacustres requiere un análisis que contemple un posible aporte no humano. Este es el caso del conjunto recuperado en el sitio Llan 50, al oeste de la laguna Llancanelo (Mendoza, Argentina). El objetivo general de este trabajo es contribuir al conocimiento de los procesos involucrados en la formación del conjunto ictioarqueológico de este sitio, con la intención de determinar si estos restos se depositaron primordialmente de forma natural o como consecuencia de la acción humana. Para ello, se comparan los patrones hallados en el registro arqueológico de Llan 50 con aquellos observados en acumulaciones naturales de peces en la misma área de estudio. Se reconocen características potenciales de los depósitos naturales en Llancanelo y se distinguen aquellos criterios válidos para diferenciar el origen de un conjunto en este contexto, los que sugerirían un origen principalmente antrópico para el conjunto arqueológico de Llan 50.

Palabras clave: Ictioarqueología, Laguna, Mendoza,Tafonomía.

\section{ABSTRACT}

The study of fish remains accumulations in archaeological contexts near to lacustrine environments requires an analysis that considers possible non-human contributions. This is the case of fish bone assemblages recovered from Llan 50 site, on the western margin of Llancanelo lagoon (Mendoza, Argentina). This paper aims to explore the processes involved in the formation of this ichthyoarchaeological assemblage, determining wether these remains were deposited naturally or as a result of human action. Consequently, the composition of bone assemblages of Llan 50 are compared with those observed for fish natural accumulations at the same study area. Natural deposits' potential characteristics from Llancanelo are recognized and some useful criteria to differentiate the assemblage origin in this context are distinguished, which would suggest mainly an anthropic origin for the archaeological assemblage Llan 50.

Key words: Ichthyoarchaeology, Lagoon, Mendoza, Taphonomy.

${ }^{i}$ CONICET- Asociación de Investigaciones Antropológicas. Bartolomé Mitre $11317^{\circ} \mathrm{G}$ - C1036AAU. Correo-e: mercedescorbat@yahoo.com.ar 


\section{INTRODUCCIÓN}

En las proximidades de la laguna Llancanelo, al sur de Mendoza, se han hallado restos de peces en contextos arqueológicos, resultando Llan 50 (Giardina et al. 20ll), sobre su margen izquierda, el sitio con la mayor evidencia ictioarqueológica registrada hasta el momento (Corbat 20II). Sin embargo, el origen de las acumulaciones de restos de peces en contextos arqueológicos próximos a las costas de ambientes lacustres requiere un análisis que contemple un posible aporte no humano (Zohar et al. 200I), especialmente porque este tipo de ecosistemas tiende a acumular huesos de peces en agregados más densos que la mayor parte de los ecosistemas terrestres (Stewart 1991). El objetivo general del trabajo es contribuir al conocimiento de los procesos involucrados en la formación de los conjuntos ictioarqueológicos de Llancanelo (figura I) -y específicamente de Llan 50- con la intención de determinar si los restos de peces en este sitio se depositaron principalmente de forma natural o como consecuencia de la acción humana. El trabajo permite incrementar nuestro conocimiento acerca de los depósitos naturales de una de las especies más representadas en los conjuntos ictioarqueológicos de Patagonia (Percichthys trucha) (Svoboda 2013) y discutir los criterios utilizados para distinguir el origen natural o cultural de este tipo de registro.



Figura 1:. Localización del área de estudio.

Figure 1: Location of the study area.

\section{ENFOQUE TEÓRICO-METODOLÓGICO}

Varios estudios han intentado discernir entre estos dos tipos de agentes -natural o antrópico- en la conformación de conjuntos de peces (Colley 1990, Wheeler y Jones 1989, Stewart 1991, Butler 1993, 1996; Zohar et al. 200I, entre otros) y han sugerido algunos criterios tentativos resumidos por Zohar et al. (200I). En este trabajo se toman en consideración algunos de estos criterios para comparar los datos obtenidos del análisis de un conjunto de peces depositado naturalmente y de un conjunto ictioarqueológico (Llan 50) con aquellos de la comunidad viva de la laguna y los cursos de agua que allí desembocan (tabla I). De esta forma pretendemos generar una primera aproximación a la composición de los conjuntos de peces en este contexto depositacional particular, previo al enterramiento, $y$ evaluar qué tan fielmente un registro de este tipo es representativo de la comunidad viva (Behrensmeyer y Dechant Boaz, 1980; Stewart, I99I; Zohar et al., 2008). Asimismo, buscamos comprender la conformación del conjunto arqueológico Llan 50 y enmarcar nuestras interpretaciones sobre el mismo.

\begin{tabular}{|l|l|}
\hline Especie & Hábitat y características generales \\
\hline $\begin{array}{l}\text { Odontesthes } \\
\text { hatcheri }\end{array}$ & $\begin{array}{l}\text { En otras lagunas aparece en las playas y } \\
\text { en el centro (Mac Donagh y Thormáhlen, } \\
\text { 1945). En Llancanelo es más frecuente en } \\
\text { las nacientes (donde aparecen los más } \\
\text { patagónico } \\
\text { (menores a I0 cm) (YPF, 2009). Suele } \\
\text { desplazarse en cardúmenes. Se destaca la } \\
\text { calidad de su carne. }\end{array}$ \\
\hline $\begin{array}{l}\text { Percichthys trucha } \\
\text { Perca criolla }\end{array}$ & $\begin{array}{l}\text { Generalmente al medio de la laguna (Mac } \\
\text { Donagh y Thormáhlen, I945; YPF, 2009). } \\
\text { ni litoral. Especie económicamente } \\
\text { importante que alcanza hasta 55 cm. }\end{array}$ \\
\hline $\begin{array}{l}\text { Jenynsia } \\
\text { multidentata } \\
\text { Madrecitas de agua }\end{array}$ & $\begin{array}{l}\text { Muy común en aguas superficiales. No } \\
\text { supera los 9,5 cm de longitud total. }\end{array}$ \\
\hline $\begin{array}{l}\text { Trichomytherus } \\
\text { areolatus } \\
\text { Bagrecito del } \\
\text { torrente }\end{array}$ & $\begin{array}{l}\text { Los juveniles preferirín aguas cercanas a } \\
\text { las orillas y se ubican entre o debajo de } \\
\text { piedras y detritus. Pueden alcanzar hasta } \\
30 \text { cm. }\end{array}$ \\
\hline $\begin{array}{l}\text { Cheirodon } \\
\text { interruptus } \\
\text { Mojarra }\end{array}$ & $\begin{array}{l}\text { Abunda en aguas mansas de arroyos y } \\
\text { ríos. Carece de valor comercial. Puede } \\
\text { alcanzar los } 6 \text { cm. }\end{array}$ \\
\hline
\end{tabular}

Tabla 1: Características de la comunidad viva de peces de la Laguna Llancanelo

Table 1: Characteristics of the living fish community at Llancanelo Lagoon. 
Las criterios aquí considerados son: a) la diversidad taxonómica: estimación de la cantidad de taxones identificados; b) la representación de partes esqueletales, previa identificación anatómica. Los porcentajes de supervivencia se calcularon dividiendo la frecuencia observada para cada sector (cráneo o postcráneo) por la frecuencia esperada. Ésta se obtiene al multiplicar el MNI de la muestra por las veces que las unidades anatómicas aparecen en el esqueleto; c) las modificaciones óseas, observando macroscópicamente la existencia de alteraciones; $d$ ) la densidad de huesos y de individuos, estimadas al dividir la cantidad de especímenes por la superficie recorrida (depósito actual) o por el volumen excavado (conjunto arqueológico) y el MNI por estos mismos valores, respectivamente; $y$ e) tamaño corporal, estimado a partir de la comparación de las vértebras, basioccipitales y faríngeos hallados con los mismos elementos de individuos de tamaño corporal conocido. La elección de los elementos responde a los de mayor representación arqueológica, exceptuando aquellos -como los otolitos- cuyo estado no permite una correcta valoración de su dimensión original, al menos macroscópicamente.

\section{PRESENTACIÓN DE LOS CONJUNTOS ANALIZADOS}

\section{Conjunto de peces actuales depositado naturalmente}

El conjunto consiste en 29 carcasas de peces depositadas naturalmente por un descenso significativo del agua de la laguna, proceso que habría comenzado un año antes de la recolección (junio $20 \mathrm{II}$ ), pero de ocurrencia cíclica. Fue recuperado en la costa norte de la laguna, en el sector inundable de playa, con suelos poco permeables y salinos. El estado de preservación es variable, tratándose generalmente de ejemplares bastante completos $y$ articulados, que incluso presentan parte del cuero y de la carne en estado seco (figura 2).

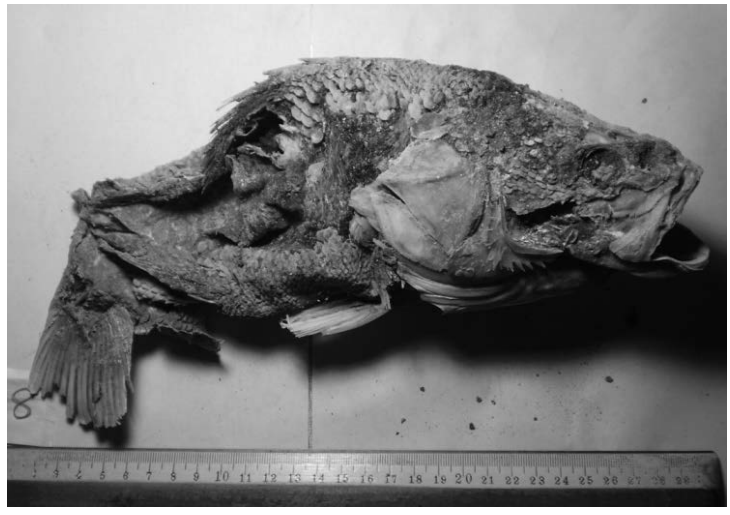

Figura 2: Carcasa de una de las percas (Percichthys trucha) depositadas naturalmente.

Figure 2: One of the perch (Percichthys trucha) carcass naturally deposited.

Conjunto ictioarqueológico de Llancanelo 50

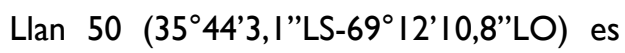
un sitio a cielo abierto ubicado aproximadamente a $7 \mathrm{~km}$ al oeste de la laguna salina de Llancanelo (I346 msnm) y a menos de $\mathrm{I} \mathrm{km}$ de los pozos de agua dulce de Carapacho. Se sitúa sobre la planicie fluviolacustre, en una zona kárstica alejada del sector de playa y cercana a los bajos salinos inundables.

Con una superficie excavada de $2 \mathrm{~m}^{2}$, se destaca la elevada cantidad de materiales líticos y restos óseos de aves pequeñas, microvertebrados y peces (Giardina et al., 20II), a lo largo de una secuencia que se extiende hasta los 3000 años AP. Estos últimos están representados por los 1105 especimenes aquí analizados.

\section{RESULTADOS}

\section{Diversidad taxonómica}

En el conjunto natural, solamente se registró una especie (Percichthys trucha) de las cuatro presentes en la laguna (tabla I). Por otro lado, la única especie identificada en el conjunto ictioarqueológico de Llancanelo es Odontesthes hatcheri, mientras que el $56 \%$ de los especímenes -mayoritariamente vértebras- permanecen taxonómicamente indeterminados (tabla 2). Esto se debe principalmente 
a problemas de identificación relacionados con la ausencia de rasgos diagnósticos en determinados elementos de los peces (Wheeler y Jones, 1989; Colley, 1990), o su desaparición -por problemas de fragmentación, erosión, termoalteración- en algunos de los restos arqueológicos. Si bien los especímenes indeterminados podrían corresponder a algunas de las otras especies, sólo se puede afirmar con certeza la presencia de pejerreyes.

\begin{tabular}{|c|c|c|c|}
\hline Elemento & Odontesthes sp. & Indet. & Total \\
\hline Basioccipital & $9(1)$ & $3(1)$ & $12(2)$ \\
\hline Ceratohial & 4 & 0 & 4 \\
\hline Faríngeo & 16 & 4 & 20 \\
\hline Opercular & । & 0 & I \\
\hline Otolito & $100(2)$ & 41 & $|4|(2)$ \\
\hline Vértebra & $356(113)$ & 507 (202) & $863(3 \mid 5)$ \\
\hline Indet. & 0 & $64(8)$ & $64(8)$ \\
\hline Total & $486(116)$ & $619(211)$ & I 105 (327) \\
\hline
\end{tabular}

Tabla 2: Abundancia anatómica y taxonómica (NISP) del conjunto ictioarqueológico Llan 50. Entre paréntesis, cantidad de restos termoalterados.

Table 2: Anatomic and taxonomic abundance (NISP) at Llan 50 ichthyoarchaeological assemblage. Quantity of burned remains in brackets.

Representación de elementos esqueléticos

Los individuos del depósito natural actual se encontraban bastantes enteros, representándose tanto su parte craneal como la postcraneal, aunque la mayoría de las carcasas presentaba el sector caudal incompleto $(65,5 \%)$ o ausente (13,8\%). Agrupando los elementos óseos por sectores, los huesos más representados en este conjunto fueron los del neurocráneo -con la mayor supervivencia (79\%)seguidos por el sector precaudal de la columna (78\%). Entre los sectores con menor supervivencia se encuentran el esqueleto apendicular (55\%), la serie orbital (49\%) y el sector caudal de la columna (47\%), lo que probablemente se relacione con la acción de carroñeros.

En el conjunto ictioarqueológico se han recuperado principalmente vértebras (sector postcraneal) y otolitos (sector craneal), predominando las primeras (tabla 2). Si analizamos el \%MNE de Llan 50, observamos que abundan los restos postcraneales $(80,4 \%)$ en clara contraposición a lo observado para los depósitos naturales recientes, cuyo segmento caudal está representado en aproximadamente un 30\% (Stewart, 1991; Stewart y Gifford-González, 1994; Butler, 1993).

\section{Modificaciones óseas}

No hemos advertido modificaciones diagnósticas en los elementos óseos del conjunto natural actual, excepto algunas fisuras y quebraduras, principalmente en preoperculares -con grietas radiales en su hoja (24\%), bordes aserrados más pronunciados (20,7\%) e incluso algunos "dientes" quebrados (14\%)-, operculares -en la porción adosada a la espina (14\%)-, etmoides (17\%) y arcos vertebrales. Aunque sin registro directo, no puede descartarse una acción carroñera por parte de algunas especies de aves.

En los especímenes de peces recuperados en el sitio Llan 50 no se han registrado marcas de corte ni señales de corrosión por ácidos estomacales. La termoalteración está presente en casi el $30 \%$ de los restos. 
Densidad huesos/individuos

En el depósito natural, la densidad de hallazgos fue relativamente baja (aproximadamente un individuo por cada $4 \mathrm{~m}^{2}$ ). Promediando la cantidad de elementos encontrados por individuo, representaría una densidad aproximada de 15 $\mathrm{NISP} / \mathrm{m}^{2}$. Sin embargo, considerando los individuos representados por mayor y menor cantidad de elementos, el rango de densidades de hallazgos por superficie se extiende desde un máximo de casi 23 $\mathrm{NISP} / \mathrm{m}^{2}$ hasta un mínimo de $2,5 \mathrm{NISP} / \mathrm{m}^{2}$. Por otro lado, en el conjunto ictioarqueológico la densidad es relativamente elevada (55I,5 especímenes por $\mathrm{m}^{3}$ ), estimándose $55, \mathrm{I} \mathrm{NISP} / \mathrm{m}^{3}$ por nivel en promedio. No obstante, la densidad varía en cada nivel, abarcando un rango de $145,5 \mathrm{NISP} / \mathrm{m}^{3}$ (nivel 3) hasta 2,5 NISP/m ${ }^{3}$ (nivel 9). A lo largo de toda la secuencia, se contabilizó un $\mathrm{MNI}=7 \mathrm{I}$ en los $2 \mathrm{~m}^{3}$.

\section{Tamaño corporal}

En el conjunto natural únicamente se recuperaron percas de 25 a $35 \mathrm{~cm}$ de largo, lo que representa un rango de tamaños relativamente acotado para la especie (tabla I). Entre los individuos de Llan 50 -de tamaño bastante uniformepredominan ampliamente aquellos de talla menor a los $22 \mathrm{~cm}$, dentro de un rango que no alcanzaría los $27 \mathrm{~cm}$. Considerando el tamaño máximo alcanzable por esta especie en este contexto $(29 \mathrm{~cm})$ y la talla promedio registrada $(20 \mathrm{~cm})$ (YPF, 2009), no se puede sostener que el rango de tamaños sea reducido.

\section{DISCUSIÓN}

La diversidad taxonómica en conjuntos culturales puede ser baja 0 alta dependiendo del método y área de pesca (Zohar et al., 200I). En nuestro caso, ambos conjuntos comparten una baja diversidad taxonómica, coincidiendo con la baja diversidad natural de Llancanelo. La especie encontrada en el depósito natural actual no refleja la fauna más litoral ni abundante de la laguna (YPF, 2009), aunque sí la de mayor tamaño. El taxón registrado en Llan 50 representa el potencialmente consumible más abundante y accesible en la zona. Si los especímenes indeterminados dieran cuenta de otra especie, esto sería un elemento a favor del origen cultural de Llan 50.

La representación de elementos esqueléticos en Llan 50 se diferencia de la registrada en depósitos naturales fósiles, donde los esqueletos aparecen relativamente completos, con una representación semejante del postcráneo y el cráneo (Stewart 1991, Zohar et al. 200I). La proporción de segmentos corporales estimada para Llan 50 puede responder a una menor preservación de los huesos craneales en el conjunto arqueológico. Sin embargo, la presencia de esqueletos incompletos coincide con lo esperado para conjuntos culturales (Stewart, 199I; Butler, 1993; Zohar et al., 200I).

Los restos ictioarqueológicos presentan pocas evidencias atribuibles a la acción humana, característica habitual en este tipo de registro (Colley, 1990; Stewart, 1991; Butler, 1996, entre otros). Las señales de combustión son tomadas como indicadores válidos de actividad humana (Stewart y Gifford-Gonzalez, 1994; Zohar et al., 200I), aunque no permitirían por sí mismas aseverar un origen antrópico (Butler, 1996; Bennett, 1999). Sin embargo, la presencia de restos calcinados hasta en los niveles más profundos y de no termoalterados en los superiores se opondrían a la posibilidad de un fuego superficial.

La alta densidad de restos de peces de Llan 50 contrasta con la de otros sitios en la misma zona (Corbat 20II) y no coincide con la situación analizada a partir del evento de varamiento de percas. Si bien se ha planteado una alta densidad en contextos naturales de pozas de marea (Van Neer, 1993, citado en Zohar et al., 200 I), el emplazamiento de Llan 50 y su contexto depositacional no lo asocian con un área sumergida.

La restricción de tamaño en Llan 50 se relacionaría con el taxón involucrado, no correspondiendo a la especie más grande disponible en los pozos actualmente: la perca. El rango restringido en el conjunto natural de perca puede estar reflejando un sesgo a favor de las especies más grandes (Stewart, 1991) y permite sostener que la baja variabilidad en los tamaños corporales no es exclusiva de los conjuntos antrópicos. Observaciones de Stewart (1991) y Van Neer (citado por Zohar et al. 200I) sobre conjuntos naturales -donde señalan tamaños exclusivamente grandes $\circ$ pequeños, respectivamente- apoyan esta idea. 


\section{CONCLUSIONES}

A partir del análisis comparativo entre el conjunto natural y arqueológico de peces se puede concluir:

I.La diversidad taxonómica no ofrece buenas condiciones para la diferenciación de depósitos naturales de los culturales -tal como se desprende también de otros estudios (Zohar et al., 200I)- , y menos aún en contextos como el de nuestra área de estudio, donde la diversidad potencial de especies es baja y existe una gran proporción de elementos indeterminados.

2. La representación diferencial de los elementos esqueléticos, las señales de combustión y la densidad de hallazgos en Llan 50 sugerirían un origen antrópico para el conjunto ictioarqueológico.

3. Los conjuntos ictiofaunísticos de Percichthys trucha con baja densidad de especímenes, incluyendo la representación de diferentes unidades anatómicas de individuos de tamaños grandes, constituyen potencialmente depósitos naturales en Llancanelo. Conocer en qué medida estos depósitos se ven transformados con el paso del tiempo o varían estacionalmente requiere de estudios específicos (experimentaciones, observaciones, excavaciones de depósitos naturales del pasado) que, si bien enriquecerían nuestras interpretaciones, exceden los alcances del presente trabajo.

4. Resta profundizar las diferencias conductuales entre la especie más representada arqueológicamente (de comportamiento gregario) y la perca.

5. La baja variabilidad de tamaños en un conjunto íctico no puede ser atribuida únicamente a pautas culturales. La evidencia del depósito natural de Llancanelo permite sostener que no todos los conjuntos con individuos de tamaño relativamente uniforme son producto de la acción humana.

\section{AGRADECIMIENTOS:}

A CONICET; A. Gil y F. Zangrando; M. Giardina, G. Neme, L. Salgán, Familia Pérez y al resto del equipo del Museo de Historia Natural de San Rafael; A L. Orquera, Martin Palma, C. Llano, Mary y la familia Franchetti. A los evaluadores.

\section{BIBLIOGRAFÍA}

Behrensmeyer, A. y D. Dechant Boaz. 1980. "The recent bones of Amboseli Park, Kenya". En Fossils in the making: Vertebrate taphonomy and paleoecology, editado por A. Behrensmeyer y A. Hill, Pp: 72-92. University of Chicago Press, Chicago.

Bennett, J. 1999. "Termal Alteration of Buried Bone". Journal of Archaeological Science 26: I-8.

Butler, V. 1993.“Natural versus cultural salmonid remains: origin of the Dalles Roadcut Bones, Columbia River, Oregon, USA“. Journal of Archaeological Science 20: I-24.

Butler, V. 1996."Tui Chub Taphonomy and the Importance of Marsh Resources in the Western Great Basin of North America“. American Antiquity 6I (4): 699-7I7.

Colley, S. 1990. "The analysis and interpretation of archaeological fish remains". En Advances in Archaeological Method and Theory 2, editado por M. Schiffer, pp: 207-253. Academic Press, San Diego.

Corbat, M. 20II. ¿Peces nativos o exóticos?. Aportes de la zooarqueología a la biología de la conservación en Laguna Llancanelo. Actas de las VIII Jornadas de Arqueología de la Patagonia, Malargüe. En prensa.

Giardina, M., G. Neme y A. Gil. 20I I. Evaluación de impacto arqueológico Proyecto exploratorio Cerro Pozo, Área de concesión Llan. R, YPF S.A. Manuscrito en posesión del autor.

Mac Donagh, E. y A. Thormáhlen. 1945 "Observaciones sobre las especies de truchas criollas". Revista del Museo de La Plata (nueva serie), IV(Zoología 27): 139-193.

Stewart, K. 1991. “Modern Fishbone Assemblages at Lake Turkana, Kenya: A Methodology to Aid in Recognition of Hominid Fish Utilization“. Journal of Archaeological Science 18: 579-603.

Stewart, K. y D. Gifford-Gonzalez. 1994. “An ethnoarchaeological contribution to identifying hominid fish processing sites". Journal of Archaeological Science 21: 237-248.

Svoboda, A. 2013. "Disponibilidad cárnica, rendimiento energético y estimación de la talla de Percichthys trucha (perca criolla) a partir de la morfometría de huesos diagnósticos y su aplicación a los conjuntos ictioarqueológicos de Patagonia central". Cuadernos de Antropología 9: 25I-266.

Wheeler, A. y A. Jones. 1989. Fishes. Cambridge University Press, Cambridge.

YPF. 2009. Estudio ambiental de la totalidad del área Llancanelo. Unidad Económica Mendoza.

Zohar, I., T. Dayan, E. Galili y E. Spanier. 200I."Fish Processing During the Early Holocene: A Taphonomic Case Study from Coastal Israel“".Journal of Archaeological Science 28: I04 I-I 053.

Zohar, I., M. Belmaker, D. Nadel, S. Gafny, M. Goren, I. Hershkovitz y T. Dayan. 2008."The living and the dead: How do taphonomic processes modify relative abundance and skeletal completeness of freshwater fish?." Palaeogeography, Palaeoclimatology, Palaeoecology 258: 292-316. 